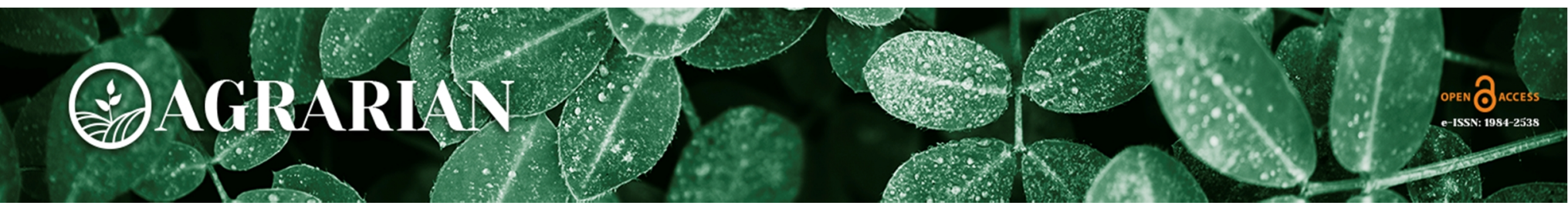

\title{
Predição das características na carne de cordeiros comerciais e pantaneiros por meio das medidas in vivo e na carcaça
}

\section{Prediction of characteristics in commercial and pantaneiros lamb meat by in vivo and carcass measurements}

Luis Gustavo Castro Alves

Universidade Federal da Grande Dourados

E-mail: gustavo353@hotmail.com

OrcID: https://orcid.org/0000-0001-6141-4066

Edson Luis de Azambuja Ribeiro Universidade Estadual de Londrina

E-mail: elar@uel.br

OrclD: https://orcid.org/0000-0001-8450-1344

Alexandre Rodrigo Mendes Fernandes Universidade Federal da Grande Dourados

E-mail: alexandrefernandes@ufg.edu.br OrcID: https://orcid.org/0000-0002-3697-9754

Fernando Miranda de Vargas Junior Universidade Federal da Grande Dourados

E-mail: fernandojunior@ufgd.edu.br

OrcID: https://orcid.org/0000-0002-3050-7107

Adriana Sathie Ozaki Hirata Universidade Federal da Grande Dourados

E-mail: adrianahirata@ufgd.edu.br OrcID: https://orcid.org/0000-0001-6087-2570

Camila Magalhães da Cunha Universidade Federal da Grande Dourados

E-mail: camis.cunha@hotmail.com OrcID: https://orcid.org/0000-0002-4297-4001

Resumo: A caracterização do sistema produtivo na ovinocultura de corte se torna atraente e competitiva com máximo de informações possíveis. Objetivou-se predizer as características dos músculos Trícpes brachii, Semimembranosus, Bíceps femoris e Longissigimus dorsi, a partir das medidas in vivo e da carcaça de cordeiros Comerciais e Pantaneiros. Foram utilizados 80 cordeiros machos, sendo 40 cordeiros Comerciais e 40 cordeiros Pantaneiros. O critério de abate foi determinado pelo peso corporal de abate de $35 \mathrm{~kg}$. Nos cordeiros Pantaneiros, a condição corporal apresentou moderada correlação com extrato etéreo no Semimembranosus $(r=0,59)$. Nas equações de regressão, as características in vivo e na carcaça apresentaram baixo coeficiente de determinação com todas as análises avaliadas nos cordeiros Comerciais. Nos cordeiros Pantaneiros as características in vivo e na carcaça apresentaram moderado coeficiente de determinação com a cor $b^{*}$ no Longissigimus dorsi $\left(R^{2}=0,57\right)$, perda de peso ao cozimento no Semimembranosus $\left(R^{2}=0,63\right)$ e alto coeficiente de determinação com a matéria mineral no Trícpes brachii $\left(R^{2}=0,70\right)$. As características in vivo e na carcaça mais representativas nas equações, nas análises instrumentais foram o peso corporal de abate, espessura de gordura subcutânea, área de olho de lombo, músculo total, relação músculo/gordura. As características in vivo e na carcaça apresentaram baixa 
correlação e não foram eficientes para predizer as características dos músculos dos cordeiros Comerciais e Pantaneiros.

\title{
Palavras-chave: Centesimal. Grupos musculares. Instrumental. Raças ovinas.
}

\begin{abstract}
The characterization of the production system and beef sheep farming becomes attractive and competitive with as much information as possible. The aim of this study was to predict the characteristics of the muscles Trícpes brachii, Semimembranosus, Bíceps femoris and Longissigimus dorsi, from the in vivo measurements and the carcass of Commercial and Pantaneiros lambs. Eighty male lambs, were evaluted, (40 animals were commercial lambs and 40 animals were pantaneiros lambs). The slaughter criterion was body weight of $35 \mathrm{~kg}$. In Pantaneiros lambs, body condition presented a moderate correlation with ether extract in Semimembranosus $(r=0.59)$. In the regression equations, the in vivo and carcass characteristics presented a low coefficient of determination in Commercial lambs. In Pantaneiros lambs, the in vivo and carcass characteristics presented a moderate coefficient of determination with the color b* in Longissigimus dorsi $(\mathrm{R} 2=$ 0.57 ), weight loss on cooking in Semimembranosus ( $R 2=0.63$ ) and high coefficient of determination with the mineral matter in Trícpes brachii $(\mathrm{R} 2=0.70)$. The most important in vivo and carcass characteristics in the equations were body weight of slaughter, subcutaneous fat thickness, ribeye area, total muscle, muscle: fat ratio. The in vivo and carcass characteristics presented low correlation and were not efficient to predict the characteristics of Commercial and Pantaneiros lambs.
\end{abstract}

Keywords: Centesimal. Instrumental. Muscles groups. Sheep breeds.

Data de recebimento: $21 / 03 / 2020$

Data de aprovação: $26 / 11 / 2020$

DOI: https://doi.org/10.30612/agrarian.v14i52.11262

\section{Introdução}

O mercado consumidor da carne, felizmente, é segmentado, o que significa que existem consumidores com interesses muito diferentes. As pesquisas contínuas sobre a qualidade da carcaça e da carne, a conscientização dos consumidores, no sentido de obter mais produtos e de forma mais acessível e idônea, ações essas, que devem contemplar todos os elos da cadeia produtiva. Com isso, há muito trabalho a ser feito, as estratégias são muitas e o trabalho bem feito é a melhor garantia para que a indústria tenha um futuro promissor (Sañudo et al., 2017; Rego et al., 2019).

Na região Centro-Oeste do Brasil a ovinocultura é uma atividade em expansão. Devido à escassez e falta de constância de carne ovina no mercado nacional. Aliado ao aumento da demanda está ocorrendo no Mato Grosso do Sul, como em outras regiões do país, um forte interesse pela criação de ovinos para produção de carne, por meio de cruzamentos entre as raças Comerciais ou àquelas adaptadas às condições locais, como é o caso dos ovinos Pantaneiros (Ferreira et al., 2020).

O cordeiro é a categoria animal que apresenta melhor eficiência produtiva e atende às exigências do consumidor (Cartaxo et al., 2017). Uma grande vantagem para o aumento da produção de carne de cordeiro no estado do Mato Grosso do Sul, reside na possibilidade da atividade poder ser rentável mesmo quando se utilizam pequenas áreas de produção, se comparado com a bovinocultura de corte (Pereira et al., 2016). Isso exige a utilização de métodos mais eficientes de terminação, como é o caso do confinamento, que reduz o tempo de terminação quando comparado a animais criados de forma extensiva (Cirne et al., 2014).

$\mathrm{O}$ conhecimento das características quantitativas e qualitativas da carne $(\mathrm{pH}$, cor, capacidade de retenção de água, perda de peso ao cozimento e maciez) e nutricionais (proteína bruta, extrato etéreo, minerais e umidade) são de fundamental importância para as indústrias que processam produtos de origem animal. A determinação desses aspectos para fins experimentais ou práticos pode estar correlacionada com as informações obtidas a partir das medidas in vivo e na carcaça, seja pelas avaliações objetivas ou subjetivas (Osório \& Osório, 2009).

Por ser apenas uma amostra da população regional de ovinos, e sabendo-se que podem ocorrer grandes variações genéticas nessas populações, e principalmente, porque o grupo chamado Comercial não Agrarian, Dourados, v. 14, n. 52, p. 244-259, 2021. 


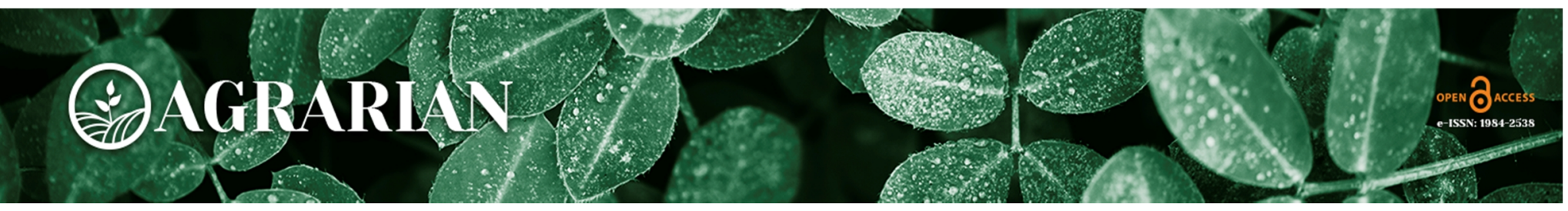

dissecação dos cortes comerciais, exceto o rabo, foram obtidos para meia carcaça os pesos de gordura total (somatório da gordura subcutânea e intermuscular), músculo total e osso. Foram calculados a relação tecidual músculo/gordura e a porção comestível, somatório da quantidade de músculo total e a gordura total.

\subsection{Análises instrumentais dos músculos}

Os músculos Tríceps brachii, Semimembranosus, Bíceps femoris, Longissimus dorsi foram seccionados, sendo retirados amostras de $2,5 \mathrm{~cm}$ de espessura, em forma de bife e sem gordura de cobertura, com o auxílio de um gabarito acrílico. As amostras foram acondicionadas em embalagens plásticas, identificadas e congeladas em freezer para posterior análises instrumentais e centesimais.

A cor do músculo foi determinada 30 minutos após a realização de um corte transversal no músculo, para a exposição da mioglobina ao oxigênio, utilizando-se colorímetro digital CR-400 Konica Minolta, calibrado no sistema CIELAB (Simões \& Ricardo, 2000).

Em seguida a avaliação da cor, foi retirada uma amostra de aproximadamente duas gramas para a determinação da capacidade de retenção de água. Utilizou-se o método da pressão segundo à técnica Cañeque \& Sañudo (2000). Para a análise de perda de água no cozimento, foi utilizado um forno elétrico préaquecido à temperatura de $170^{\circ} \mathrm{C}$ e as amostras foram assadas até atingir $70^{\circ} \mathrm{C}$ no seu centro geométrico, monitorando com termômetro digital. Os pesos dos bifes antes e depois da cocção foram utilizados para os cálculos das perdas por cozimento.

Após resfriamento a temperatura ambiente dos bifes assados, com vazador de alumínio adpatdo de $1,3 \mathrm{~cm}$ de diâmetro retiraram-se subamostras no sentido longitudinal das fibras muscular para determinar a força de cisalhamento (FC) em texturômetro, aclopado à lâmina Warner Bratzler Force, expressa em kgf, conforme a metodologia proposta por Osório et al. (2008).

\subsection{Análise centesimal}

A análise centesimal foi realizada no Laboratório de Nutrição Animal do Centro de Ciências Agrárias da Universidade Estadual de Londrina (UEL). Após o descongelamento dos músculos, retirou-se a gordura externa com o auxílio do bisturi e a amostra foi triturada utilizando-se um processador de alimentos para a homogeneização. Em seguida, as amostras em duplicata foram secas em estufa a $105^{\circ} \mathrm{C}$, por 24 horas. As mesmas foram moídas para determinação de proteína bruta, extrato etéreo e matéria mineral pelos métodos 950.46; 981.10; 960.39 e 920.153, respectivamente, descritos pela AOAC (2005).

\subsection{Análise estatística}

Para as análises estatísticas foram utilizadas primeiramente uma análise de correlação de Pearson, e posteriormente os dados foram submetidos à análise de regressão linear múltipla por meio do procedimento Stepwise. As análises foram realizadas pelo programa estatístico RStudio versão 2.15.0.

A equação de regressão múltipla é descrita a seguir: $Y=b 0+b 1 \times 1+\ldots+b j x j+e$. Onde $Y$ são as variáveis dependentes e os X's são as variáveis independentes, b0 é o intercepto da regressão, b1 até bj são os coeficientes da regressão e o valor de "e" o erro aleatório da regressão.

\section{Resultados e discussões}

\subsection{Análise descritiva}

A análise descritiva das variáveis independentes, medidas in vivo e na carcaça e as variáveis dependentes, análises instrumentais e centesimais dos músculos Tríceps brachii, Semimembranosus, Bíceps femoris, Longissimus dorsi estão nas Tabelas 1, 2 e 3.

\subsection{Análise de correlação}


Nas tabelas 4, 5 e 6 estão apresentadas as correlações estimadas entre as características in vivo e na carcaça com as análises instrumentais e centesimais dos músculos Tríceps brachii, Semimembranosus, Bíceps femoris, Longissimus dorsi. Essas características nos músculos dos cordeiros Comerciais apresentaram baixa correlação, isto pode ser justificado por apresentarem maior heterogeneidade no lote $\mathrm{e}$ por não possuírem padrão racial específico.

Nos cordeiros Pantaneiros, apresentaram moderada correlação, a condição corporal com a cor $b^{*}$ no Longissimus dorsi $(r=-0,50)$, umidade no Semimembranosus $(r=0,52)$ e pH no Longissimus dorsi $(r=-0,52)$.

O extrato etéreo foi correlacionado com as medidas que avaliam a distribuição de gordura subcutânea na carcaça, como a condição corporal $(r=0,59)$ e estado de engorduramento $(r=0,50)$ no Semimembranosus. E do Tríceps brachii com a espessura de gordura subcutânea $(r=0,50)$. Santos et al. (2008) afirmaram que o extrato etéreo e a energia estão correlacionados, ou seja, com a proximidade da maturidade do ovino em crescimento, à medida que o peso corporal se eleva, há um aumento na proporção de gordura. Em raças mais rústicas, como é o caso dos ovinos Pantaneiros, o ímpeto de deposição das gorduras na carcaça é diferente, podem apresentar maior deposição de gordura interna (visceral), fato esse justificado por seu processo evolutivo e do efeito da reserva energética (Osório et al., 2014).

O índice de compacidade da carcaça foi correlacionado com a perda de peso ao cozimento no Tríceps brachii $(r=0,54)$. A gordura total correlacionada com a perda de peso ao cozimento no Semimembranosus ( $r$ $=-0,51)$. Sañudo et al. (1997) ressaltaram a influência da gordura total sobre a qualidade da carne, maiores níveis de gordura intra e intermuscular conduzem a menores perda de peso ao cozimento e, consequentemente, a obtenção de carnes mais suculentas, visto que a gordura presente na carne atua como barreira contra a perda de umidade.

O músculo total com cor $a^{*}$ no Semimembranosus $(r=-0,54)$ e com cor $b^{*}$ no Semimembranosus $(r=-$ 0,52 ). Músculos mais ativos e que exercem mais força, como os músculos do dianteiro dos animais, responsáveis pela sustentação e locomoção, tendem a influenciar, por exemplo, na cor, onde são mais ricos em fibras vermelhas (Gomide et al., 2013).

A relação músculo/gordura com a cor $a^{*}$ no Semimembranosus $(r=-0,56)$, capacidade de retenção de água no Semimembranosus $(r=-0,50)$, perda de peso ao cozimento no Semimembranosus $(r=0,55)$ e a umidade no Tríceps brachii $(r=-0,60)$. A porção comestível com a cor $b^{*}$ no Semimembranosus $(r=-0,51)$ e a área de olho de lombo apresentou alta correlação com a umidade no Tríceps brachii $(r=-0,62)$. Pode inferir que as quantidades de músculo e gordura tiveram participação nas análises dos diferentes músculos analisados, com isso interferiram nas características quantitativas e qualitativas.

Dentre os músculos estudados, o Semimembranosus foi o que apresentou maior correlação com as características avaliadas, isto pode estar relacionado a sua estrutura e funcionalidade, por esta razão, deve ser melhor explorado já que é um músculo com grande representatividade e um dos que configura o índice de musculosidade do pernil.

\subsection{Análise de regressão}

As equações de regressão múltipla estão nas Tabelas 7, 8 e 9. Nos cordeiros Comerciais, (Tabela 7), de modo geral, as características in vivo e na carcaça foram pouco relacionadas e participativas em predizer as análises qualitativas dos músculos Tríceps brachii, Bíceps femoris, Semimembranosus, Longissimus dorsi, possivelmente em decorrência da heterogeneidade do lote.

Nos cordeiros Pantaneiros, (Tabela 8), apenas o modelo da perda de peso ao cozimento do Bíceps femoris não foi significativo. As características in vivo: peso corporal de abate e condição corporal e as características na carcaça: conformação, estado de engorduramento, espessura de gordura subcutânea, área de olho de lombo, músculo total, estado de engorduramento, espessura de gordura subcutânea e músculo total apresentaram associação com a cor $b^{*}$ no Semimembranosus $\left(R^{2}=0,50\right)$ e no Longissimus dorsi $\left(R^{2}=\right.$ $0,57)$. E essas características com a relação músculo/gordura tiveram associação com a perda de peso ao cozimento no Semimembranosus $\left(R^{2}=0,63\right)$.

As equações tiveram a inserção de até sete variáveis independentes, sendo a porção comestível única característica a não entrar no modelo. As características mais participativas: conformação, peso corporal de abate, condição corporal, área de olho de lombo, espessura de gordura subcutânea e estado de engorduramento. De um modo geral, houve a participação de $91 \%$ das variáveis e, a predominância de $63 \%$

Agrarian, Dourados, v. 14, n. 52, p. 244-259, 2021. 
dessas variáveis nos modelos, sendo estas, características importantes na constituição da composição tecidual da carcaça.

$\mathrm{Na}$ Tabela 9, as características peso corporal de abate, condição corporal, espessura de gordura subcutânea, índice de compacidade da carcaça e relação músculo/gordura com a umidade no Tríceps brachii $\left(R^{2}=0,70\right)$ nos cordeiros Pantaneiros. Dentre estas características, Cruz et al. (2016) ressaltaram que a umidade tem grande influência na qualidade da carne, como na sua suculência, textura, cor e sabor e nos processos que a mesma irá sofrer posteriormente.

As equações tiveram a inserção de até oito variáveis independentes para ambos os grupos avaliados, sendo a porção comestível única característica a não entrar no modelo. As características mais participativas: espessura de gordura subcutânea, estado de engorduramento, área de olho de lombo e músculo total. Houve a participação de $91 \%$ das variáveis e, a predominância de $72 \%$ dessas variáveis nos modelos. Vale ressaltar que esses resultados podem ter ocorrido, em decorrência, da funcionalidade das características qualitativas avaliadas e do tipo de atividade exercida por esses músculos.

Menezes et al. (2015) elaboraram modelos para predição da composição física e química corporal em ovinos a partir de medidas obtidas da seção da $9^{a}$ à $11^{a}$ costelas ou da $12^{a}$ costela e concluíram que é possível gerar modelos de predição de componentes físicos e químicos corporais a partir dessas informações. Em contrapartida, Lambe et al. (2009) avaliaram as características post-mortem sobre as características qualitativas na carne e observaram que tiveram pouca participação na predição, como na força de cisalhamento dos músculos Longissimus dorsi e Semimembranoso, com poucas precisões para sugerir que possam ser úteis em um nível comercial. Essa última informação corrobora com os resultados obtidos no presente estudo, reforçando que as características in vivo e na carcaça não apresentaram efeito decisivo para prever as características na carne.

Tabela 1. Análise descritiva das características in vivo e na carcaça de cordeiros Comerciais e Pantaneiros.

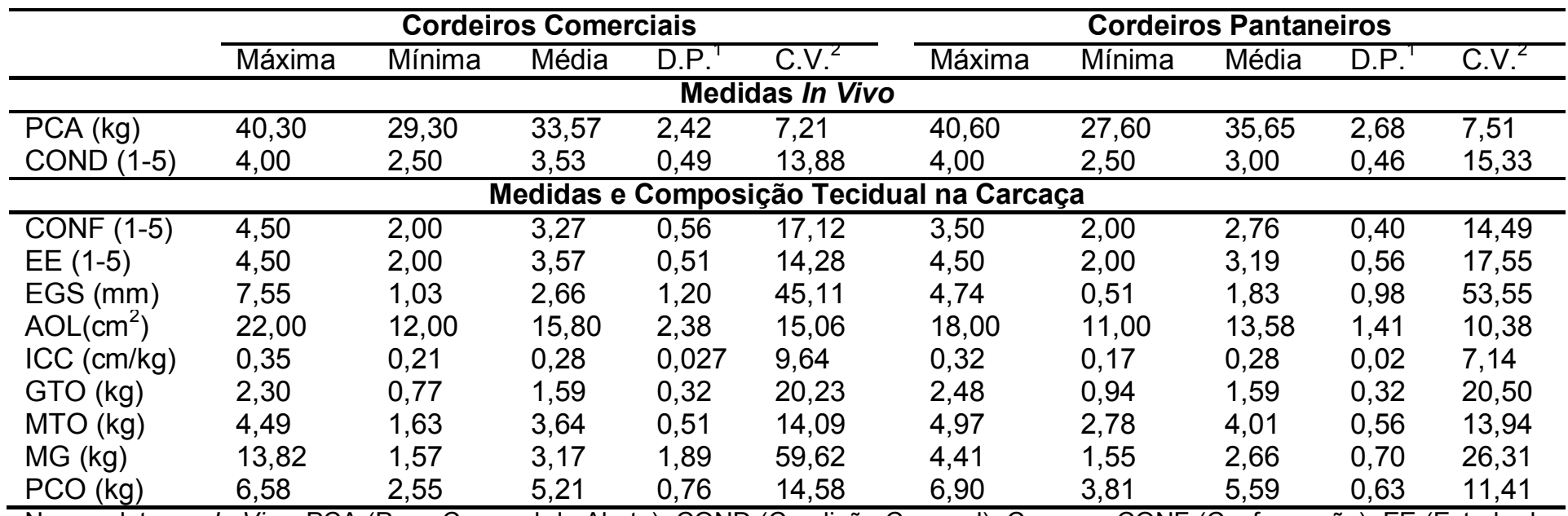

Nomenclaturas: In Vivo: PCA (Peso Corporal de Abate); COND (Condição Corporal). Carcaça: CONF (Conformação); EE (Estado de Engorduramento); EGS (Espessura de Gordura Subcutânea); AOL (Área de Olho de Lombo); ICC (Índice de Compacidade da Carcaça); GTO (Gordura Total - gordura subcutânea e intermuscular dos cortes comerciais); MTO (Músculo Total); MG (Relação Músculo/Gordura Total); PCO (Porção Comestível - somatório do peso de músculo e gordura total); ${ }^{1}$ D.P - Desvio Padrão; ${ }^{2}$ C.V. Coeficiente de Variação (\%).

Tabela 2. Análise descritiva das análises instrumentais dos músculos Longissimus dorsi, Semimembranosus, Bíceps femoris, Triceps brachii, dos cortes de cordeiros Comerciais e Pantaneiros.

\begin{tabular}{|c|c|c|c|c|c|c|c|c|c|c|}
\hline \multirow[b]{2}{*}{ Músculos } & \multicolumn{5}{|c|}{ Cordeiros Comerciais } & \multicolumn{5}{|c|}{ Cordeiros Pantaneiros } \\
\hline & Máxima & Mínima & Média & D. P. ${ }^{\top}$ & C.V. ${ }^{2}$ & Máxima & Mínima & Média & D.P. ${ }^{\prime}$ & C. V. ${ }^{2}$ \\
\hline \multicolumn{11}{|c|}{ pH final } \\
\hline Longissimus dorsi & 6,16 & 5,47 & 5,82 & 0,18 & 3,09 & 6,10 & 5,41 & 5,65 & 0,17 & 3,00 \\
\hline Semimembranosus & 6,35 & 5,51 & 5,76 & 0,19 & 3,29 & 6,08 & 5,38 & 5,69 & 0,18 & 3,16 \\
\hline Trícpes brachii & 6,40 & 5,50 & 5,89 & 0,15 & 2,54 & 6,04 & 5,60 & 5,82 & 0,11 & 1,89 \\
\hline
\end{tabular}


Cor $L^{*}$

\begin{tabular}{|c|c|c|c|c|c|c|c|c|c|c|}
\hline Longissimus dorsi & 41,06 & 28,18 & 33,87 & 2,84 & 8,38 & 45,02 & 32,82 & 40,47 & 2,60 & 6,47 \\
\hline Semimembranosus & 42,86 & 24,87 & 34,51 & 2,92 & 8,46 & 48,87 & 31,37 & 40,22 & 3,73 & 9,27 \\
\hline Trícpes brachii & 41,19 & 32,59 & 36,96 & 2,05 & 5,54 & 47,86 & 36,85 & 42,24 & 2,93 & 6,93 \\
\hline \multicolumn{11}{|c|}{ Cor $a^{*}$} \\
\hline Longissimus dorsi & 20,75 & 15,18 & 17,39 & 1,39 & 7,99 & 25,52 & 15,14 & 22,03 & 2,55 & 11,57 \\
\hline Semimembranosus & 21,43 & 14,49 & 17,59 & 1,65 & 9,38 & 27,27 & 14,73 & 22,48 & 2,85 & 12,67 \\
\hline Trícpes brachii & 22,57 & 10,88 & 16,43 & 2,48 & 15,09 & 24,71 & 11,88 & 20,70 & 3,31 & 15,99 \\
\hline \multicolumn{11}{|c|}{ Cor $b^{*}$} \\
\hline Longissimus dorsi & 16,16 & $\overline{3,44}$ & 6,52 & 2,28 & 34,96 & 11,23 & 4,83 & 7,67 & 1,79 & 23,33 \\
\hline Semimembranosus & 11,43 & 2,74 & 7,20 & 2,13 & 29,58 & 13,06 & 4,25 & 7,71 & 2,47 & 32,03 \\
\hline Trícpes brachii & 10,59 & 1,80 & 5,69 & 2,09 & 36,76 & 9,82 & 2,23 & 6,80 & 1,85 & 27,20 \\
\hline \multicolumn{11}{|c|}{ Capacidade Retenção Água } \\
\hline Longissimus dorsi & 89,50 & 61,34 & 72,80 & 4,99 & 6,85 & 91,30 & 73,71 & 83,16 & 4,46 & 5,36 \\
\hline Bíceps femoris & 86,55 & 66,55 & 76,10 & 4,37 & 5,74 & 92,00 & 74,25 & 85,03 & 4,33 & 5,09 \\
\hline Semimembranosus & 82,00 & 66,58 & 73,55 & 4,11 & 5,58 & 88,35 & 74,72 & 84,30 & 3,36 & 3,96 \\
\hline Trícpes brachii & 86,50 & 66,98 & 76,08 & 4,65 & 6,11 & 97,72 & 54,57 & 87,31 & 8,98 & 10,28 \\
\hline \multicolumn{11}{|c|}{ Perda de Peso ao Cozimento } \\
\hline Longissimus dorsi & 45,81 & 24,43 & 37,13 & 4,54 & 12,22 & 42,07 & 12,93 & 28,85 & 6,05 & 20,97 \\
\hline Bíceps femoris & 47,81 & 27,13 & 33,64 & 4,31 & 12,81 & 40,15 & 16,58 & 31,71 & 5,13 & 16,17 \\
\hline Semimembranosus & 45,52 & 11,14 & 37,06 & 7,68 & 20,72 & 37,42 & 23,16 & 29,45 & 4,21 & 14,29 \\
\hline Trícpes brachii & 45,58 & 24,40 & 37,37 & 4,33 & 11,58 & 47,67 & 18,18 & 30,56 & 6,58 & 21,53 \\
\hline \multicolumn{11}{|c|}{ Força de Cisalhamento } \\
\hline Longissimus dorsi & 7,89 & 1,46 & 4,65 & 1,72 & 36,98 & 6,86 & 1,65 & 3,57 & 1,23 & 34,45 \\
\hline Bíceps femoris & 5,46 & 1,75 & 3,39 & 0,91 & 26,84 & 3,06 & 1,74 & 2,24 & 0,40 & 17,85 \\
\hline Semimembranosus & 8,28 & 3,46 & 5,65 & 1,08 & 19,11 & 5,74 & 1,77 & 3,26 & 0,90 & 27,60 \\
\hline Trícpes brachii & 4,91 & 1,69 & 2,76 & 0,69 & 25,00 & 5,35 & 2,28 & 3,32 & 0,73 & 21,98 \\
\hline
\end{tabular}

Músculos: Longissimus dorsi (Lombo); Semimembranosus e Bíceps femoris (Pernil); Tríceps brachii (Paleta) 'D.P - Desvio Padrão;

${ }^{2}$ C.V. - Coeficiente de Variação (\%).

Tabela 3. Análise descritiva das análises centesimais dos músculos Longissimus dorsi, Semimembranosus, Bíceps femoris, Triceps brachii, dos cortes de cordeiros Comerciais e Pantaneiros.

\begin{tabular}{|c|c|c|c|c|c|c|c|c|c|c|}
\hline \multirow[b]{2}{*}{ Músculos } & \multicolumn{5}{|c|}{ Cordeiros Comerciais } & \multicolumn{5}{|c|}{ Cordeiros Pantaneiros } \\
\hline & Máxima & Mínima & Média & D.P. & C.V. ${ }^{2}$ & Máxima & Mínima & Média & D.P. & C.V. ${ }^{2}$ \\
\hline \multicolumn{11}{|c|}{ Proteína Bruta } \\
\hline Longissimus dorsi & 23,04 & 18,00 & 20,26 & 1,19 & 5,87 & 22,90 & 14,83 & 19,26 & 1,66 & 8,61 \\
\hline Bíceps femoris & 20,87 & 17,24 & 19,39 & 0,83 & 4,28 & 21,87 & 15,22 & 18,39 & 1,55 & 8,42 \\
\hline Semimembranosus & 23,45 & 16,48 & 19,36 & 1,45 & 7,48 & 25,42 & 13,38 & 19,49 & 2,46 & 12,62 \\
\hline Trícpes brachii & 20,45 & 15,87 & 18,15 & 1,07 & 5,89 & 20,55 & 16,11 & 17,96 & 1,01 & 5,62 \\
\hline \multicolumn{11}{|c|}{ Extrato Etéreo } \\
\hline Longissimus dorsi & 8,48 & 1,40 & 4,94 & 1,47 & 29,75 & 9,87 & 1,54 & 4,45 & 2,05 & $\overline{46,06}$ \\
\hline Bíceps femoris & 6,25 & 1,09 & 3,11 & 1,01 & 32,47 & 9,47 & 2,27 & 5,26 & 1,47 & 27,94 \\
\hline Semimembranosus & 5,55 & 1,56 & 3,09 & 0,94 & 30,42 & 8,55 & 0,78 & 4,34 & 2,36 & 54,37 \\
\hline Trícpes brachii & 9,67 & 1,14 & 5,60 & 1,93 & 34,46 & 11,83 & 3,51 & 5,61 & 1,62 & 28,87 \\
\hline \multicolumn{11}{|c|}{ Umidade } \\
\hline Longissimus dorsi & 75,31 & 69,45 & 72,49 & 1,55 & 2,13 & 77,60 & 69,34 & 74,51 & 1,74 & 2,33 \\
\hline Bíceps femoris & 75,40 & 70,24 & 73,41 & 1,11 & 1,51 & 78,91 & 68,13 & 75,62 & 2,07 & 2,73 \\
\hline Semimembranosus & 87,76 & 70,75 & 76,60 & 3,12 & 4,07 & 82,75 & 69,34 & 75,87 & 3,04 & 4,00 \\
\hline Trícpes brachii & 76,64 & 69,45 & 72,84 & 1,73 & 2,37 & 82,75 & 67,26 & 76,60 & 3,21 & 4,19 \\
\hline \multicolumn{11}{|c|}{ Matéria Mineral } \\
\hline Longissimus dorsi & 1,10 & 0,71 & 0,90 & 0,09 & 10,00 & 3,17 & 1,02 & 1,40 & 0,39 & 27,85 \\
\hline Bíceps femoris & 1,19 & 0,82 & 0,98 & 0,09 & 9,18 & 3,17 & 0,93 & 1,30 & 0,35 & 26,92 \\
\hline
\end{tabular}

Agrarian, Dourados, v. 14, n. 52, p. 244-259, 2021. 


\section{Pagrarian}

Semimembranosus

Trícpes brachii
$0,19 \quad 22,35$

$0,90 \quad 0,08 \quad 8,88$
1,88

1,40
0,78

0,98
1,26

0,21

16,66

1,13

${ }^{2}$ C.V. - Coeficiente de Variação (\%).

Tabela 4. Correlação entre as características in vivo e na carcaça com as análises instrumentais dos músculos de cordeiros Comerciais.

\begin{tabular}{|c|c|c|c|c|c|c|c|c|c|c|c|}
\hline Músculos & $\overline{P C A}$ & COND & CONF & EE & EGS & $\overline{A O L}$ & ICC & GTO & MTO & $M G$ & $\mathrm{PCO}$ \\
\hline \multicolumn{12}{|c|}{ pH Final } \\
\hline Longissimus dorsi & 0,30 & $0,33^{*}$ & $\overline{0,06}$ & 0,03 & $-0,16$ & 0,13 & $\overline{0,11}$ & 0,00 & 0,01 & $-0,03$ & $\overline{0,01}$ \\
\hline Semimembranosus & $-0,17$ & 0,07 & $-0,03$ & - & $-0,15$ & $-0,12$ & $-0,31$ & $-0,26$ & $-0,20$ & 0,12 & $-0,26$ \\
\hline Trícpes brachii & 0,00 & 0,08 & $-0,16$ & $-0,11$ & - & 0,05 & $-0,16$ & $-0,14$ & 0,07 & 0,14 & $-0,03$ \\
\hline \multicolumn{12}{|c|}{ Cor $L^{*}$} \\
\hline Longissimus dorsi & $-0,29$ & $-0,36^{*}$ & $-0,12$ & $-0,13$ & $-0,29$ & $-0,31$ & $-0,22$ & $-0,18$ & $-0,16$ & $-0,09$ & $-0,20$ \\
\hline Semimembranosus & - & $-0,29$ & $-0,13$ & $-0,06$ & $-0,26$ & $-0,31$ & - & $-0,15$ & $-0,03$ & 0,10 & $-0,10$ \\
\hline Trícpes brachii & 0,01 & $-0,30$ & $-0,14$ & $-0,21$ & - & $-0,30$ & $-0,28$ & $-0,26$ & 0,05 & 0,27 & $-0,10$ \\
\hline \multicolumn{12}{|c|}{ Cor $\mathbf{a}^{*}$} \\
\hline Longissimus dorsi & $\overline{0,02}$ & $-0,07$ & $-0,10$ & 0,04 & $-0,05$ & $-0,01$ & $\overline{0,00}$ & $-0,12$ & $-0,08$ & 0,04 & $-0,12$ \\
\hline Semimembranosus & 0,18 & 0,17 & $-0,15$ & $-0,20$ & - & 0,12 & $-0,14$ & $-0,25$ & 0,00 & 0,18 & $-0,13$ \\
\hline Trícpes brachii & $-0,03$ & 0,13 & 0,09 & $-0,03$ & $-0,01$ & 0,12 & 0,12 & 0,04 & 0,09 & $-0,05$ & 0,08 \\
\hline \multicolumn{12}{|c|}{ Cor $b^{*}$} \\
\hline Longissimus dorsi & $-0,28$ & $-0,04$ & $-0,14$ & $-0,07$ & $-0,17$ & 0,09 & $-0,11$ & $-0,12$ & $-0,06$ & 0,04 & $-0,10$ \\
\hline Semimembranosus & 0,07 & 0,17 & $-0,16$ & $-0,24$ & - & 0,06 & $-0,29$ & $-0,22$ & 0,18 & 0,26 & 0,00 \\
\hline Trícpes brachii & $-0,15$ & 0,08 & $-0,12$ & $-0,15$ & $-0,09$ & 0,04 & $-0,09$ & $-0,06$ & 0,05 & 0,03 & 0,01 \\
\hline \multicolumn{12}{|c|}{ Capacidade Retenção Água } \\
\hline Longissimus dorsi & 0,22 & 0,09 & 0,12 & 0,18 & 0,12 & 0,09 & 0,21 & 0,18 & 0,16 & $-0,13$ & 0,20 \\
\hline Bíceps femoris & $-0,11$ & $-0,24$ & $-0,22$ & $-0,06$ & $-0,05$ & $-0,07$ & $-0,12$ & $-0,26$ & $-0,12$ & 0,20 & $-0,22$ \\
\hline Semimembranosus & $-0,16$ & $-0,19$ & $-0,16$ & $-0,08$ & 0,20 & $-0,10$ & 0,07 & $-0,12$ & $-0,31$ & 0,01 & $-0,26$ \\
\hline Trícpes brachii & $-0,11$ & 0,03 & 0,15 & 0,17 & 0,17 & 0,04 & 0,24 & 0,13 & 0,08 & $-0,01$ & 0,12 \\
\hline \multicolumn{12}{|c|}{ Perda de Peso ao Cozimento } \\
\hline Longissimus dorsi & $-0,15$ & $-0,13$ & $-0,12$ & $-0,10$ & $-0,13$ & $-0,24$ & $-0,23$ & $-0,07$ & 0,19 & 0,19 & 0,09 \\
\hline Bíceps femoris & $-0,13$ & $-0,11$ & 0,19 & $-0,03$ & 0,12 & 0,00 & $-0,03$ & 0,10 & 0,12 & $-0,08$ & 0,13 \\
\hline Semimembranosus & $-0,22$ & $-0,34^{*}$ & $-0,08$ & $-0,10$ & 0,22 & $-0,11$ & $-0,12$ & $-0,03$ & $-0,21$ & 0,03 & $-0,16$ \\
\hline Trícpes brachii & 0,01 & $-0,09$ & 0,12 & $-0,18$ & $-0,26$ & 0,10 & $-0,23$ & $-0,12$ & $0,37^{*}$ & $0,32^{*}$ & 0,18 \\
\hline \multicolumn{12}{|c|}{ Força de Cisalhamento } \\
\hline Longissimus dorsi & $-0,05$ & 0,19 & 0,11 & 0,02 & 0,12 & 0,04 & $-0,02$ & 0,20 & $0,35^{*}$ & 0,03 & $0,33^{*}$ \\
\hline Bíceps femoris & 0,04 & 0,21 & $-0,20$ & $-0,16$ & $-0,13$ & $-0,08$ & $-0,15$ & $-0,14$ & 0,12 & 0,14 & 0,00 \\
\hline Semimembranosus & $-0,22$ & $-0,20$ & 0,09 & $-0,6$ & 0,09 & $-0,08$ & $-0,16$ & $-0,03$ & $-0,09$ & 0,05 & $-0,07$ \\
\hline Trícpes brachii & $-0,11$ & $-0,01$ & 0,14 & 0,12 & 0,07 & 0,03 & 0,13 & 0,21 & $-0,04$ & $-0,37^{*}$ & 0,08 \\
\hline
\end{tabular}

Músculos: Longissimus dorsi (Lombo); Semimembranosus e Bíceps femoris (Pernil); Tríceps brachii (Paleta). In Vivo: PCA (Peso Corporal de Abate); COND (Condição Corporal). Carcaça: CONF (Conformação); EE (Estado de Engorduramento); EGS (Espessura de Gordura Subcutânea); AOL (Área de Olho de Lombo); ICC (Índice de Compacidade da Carcaça); GTO (Gordura Total - gordura subcutânea e intermuscular); MTO (Músculo Total); MG (Relação Músculo/Gordura Total); PCO (Porção Comestível - peso de músculo e gordura total). $\left({ }^{*} \mathrm{P}<0,05\right)$.

Tabela 5. Correlação entre as características in vivo e na carcaça com as análises instrumentais dos músculos de cordeiros Pantaneiros.

\begin{tabular}{lccccccccccc}
\hline & PC & CONDC & CONF & EE & EGS & AOL & ICC & GTO & MTO & MG & PCO \\
\hline & \multicolumn{10}{c}{ pH Final } \\
\hline Longissimus dorsi & - & $-0,52^{*}$ & $-0,11$ & $-0,34^{*}$ & $-0,07$ & $-0,17$ & $-0,12$ & $-0,24$ & $-0,31$ & 0,03 & $-0,39^{*}$ \\
Semimembranosus & - & $-0,44^{*}$ & $-0,07$ & $-0,25$ & 0,06 & $-0,20$ & $-0,06$ & $-0,10$ & $-0,32$ & $-0,10$ & $-0,33^{*}$ \\
Trícpes brachii & - & $-0,31$ & $-0,04$ & $-0,13$ & $-0,01$ & $-0,05$ & $-0,22$ & $-0,16$ & $-0,12$ & 0,07 & $-0,19$ \\
\hline & \multicolumn{10}{c}{ Cor L* } \\
\hline & 0,04 & 0,07 & 0,08 & 0,10 & 0,00 & $-0,25$ & $0,37^{*}$ & 0,28 & $-0,36^{*}$ & $-0,47^{*}$ & $-0,17$
\end{tabular}

Agrarian, Dourados, v. 14, n. 52, p. 244-259, 2021. 
idackaran

\begin{tabular}{|c|c|c|c|c|c|c|c|c|c|c|c|}
\hline Semimembranosus & 0,04 & 0,16 & 0,05 & 0,09 & 0,02 & $-0,37^{*}$ & 0,20 & 0,26 & $-0,32^{*}$ & $-0,45^{*}$ & $-0,15$ \\
\hline Trícpes brachii & 0,01 & 0,21 & $-0,09$ & 0,10 & 0,02 & $-0,15$ & 0,11 & 0,16 & $-0,02$ & $-0,12$ & 0,07 \\
\hline \multicolumn{12}{|c|}{ Cor $\mathrm{a}^{*}$} \\
\hline Longissimus dorsi & 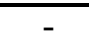 & 0,05 & 0,23 & 0,20 & $-0,23$ & $-0,29$ & 0,17 & $0,33^{*}$ & $-0,36^{*}$ & $-0,51^{*}$ & $-0,14$ \\
\hline Semimembranosus & - & $-0,13$ & 0,05 & 0,05 & $-0,11$ & $-0,23$ & 0,29 & 0,30 & $-0,54^{*}$ & $-0,56^{*}$ & $-0,32^{*}$ \\
\hline Trícpes brachii & 0,10 & 0,13 & $0,34^{*}$ & $0,39^{*}$ & $-0,02$ & $-0,01$ & 0,29 & $0,38^{*}$ & $-0,17$ & $-0,45^{*}$ & 0,04 \\
\hline \multicolumn{12}{|c|}{ Cor b* } \\
\hline Longissimus dorsi & - & $-0,50^{*}$ & $\overline{0,01}$ & $-0,37^{*}$ & $-0,22$ & $-0,08$ & $0,36^{*}$ & $-0,14$ & $-0,46^{*}$ & $-0,18$ & $-0,47^{*}$ \\
\hline Semimembranosus & - & $-0,46^{*}$ & $-0,22$ & $-0,41$ & $-0,08$ & $-0,20$ & 0,27 & $-0,10$ & $-0,52^{*}$ & $-0,20$ & $-0,51^{*}$ \\
\hline Trícpes brachii & 0,02 & $-0,17$ & 0,14 & 0,20 & 0,06 & 0,24 & 0,33 & 0,29 & $-0,10$ & $-0,35^{*}$ & 0,06 \\
\hline \multicolumn{12}{|c|}{ Capacidade de Retenção de Agua } \\
\hline Longissimus dorsi & - & 0,06 & $-0,18$ & 0,09 & $-0,22$ & 0,25 & $-0,16$ & 0,29 & $-0,28$ & $-0,41$ & $-0,09$ \\
\hline Bíceps femoris & - & $-0,04$ & $-0,08$ & 0,23 & $-0,34^{*}$ & $0,30^{*}$ & $-0,23$ & $0,31^{*}$ & $-0,41^{*}$ & $-0,49^{*}$ & $-0,20$ \\
\hline Semimembranosus & 0,01 & 0,06 & 0,02 & 0,06 & $-0,48^{*}$ & 0,23 & $-0,04$ & $0,38^{*}$ & $-0,28$ & $-0,50^{*}$ & $-0,05$ \\
\hline Trícpes brachii & - & 0,00 & 0,06 & 0,17 & $-0,18$ & 0,02 & $-0,15$ & 0,29 & $-0,37^{*}$ & $-0,44^{*}$ & $-0,18$ \\
\hline \multicolumn{12}{|c|}{ Perda de Peso ao Cozimento } \\
\hline Longissimus dorsi & - & $-0,33^{*}$ & $\overline{0,03}$ & $-0,38^{*}$ & 0,00 & $-0,06$ & $-0,23$ & $-0,49^{*}$ & 0,03 & $0,35^{*}$ & $-0,23$ \\
\hline Bíceps femoris & - & $-0,20$ & $-0,02$ & $-0,15$ & $-0,02$ & 0,04 & $-0,18$ & $-0,20$ & $-0,10$ & 0,16 & $-0,19$ \\
\hline Semimembranosus & 0,06 & $-0,15$ & 0,18 & $-0,38^{*}$ & 0,26 & $-0,19$ & $-0,03$ & $-0,51^{*}$ & 0,19 & $0,55^{*}$ & $-0,09$ \\
\hline Trícpes brachii & 0,23 & $-0,19$ & $-0,06$ & $-0,29$ & 0,14 & $-0,13$ & $0,54^{*}$ & $-0,08$ & 0,24 & 0,20 & 0,17 \\
\hline \multicolumn{12}{|c|}{ Força de Cisalhamento } \\
\hline Longissimus dorsi & - & $-0,22$ & $\overline{0,05}$ & $-0,22$ & $-0,13$ & 0,20 & $-0,14$ & $-0,02$ & $-0,30$ & $-0,19$ & $-0,28$ \\
\hline Bíceps femoris & 0,09 & $-0,04$ & $-0,08$ & $-0,18$ & $-0,02$ & 0,10 & 0,06 & $-0,06$ & 0,07 & 0,06 & 0,03 \\
\hline Semimembranosus & 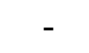 & $-0,05$ & $-0,01$ & 0,12 & 0,09 & $-0,03$ & $-0,26$ & 0,23 & $-0,09$ & $-0,25$ & 0,04 \\
\hline Trícpes brachii & 0,01 & $0,37^{*}$ & $-0,03$ & $0,39^{*}$ & $-0,10$ & 0,00 & 0,00 & $0,35^{*}$ & $-0,17$ & $-0,33$ & 0,03 \\
\hline
\end{tabular}

Músculos: Longissimus dorsi (Lombo); Semimembranosus e Bíceps femoris (Pernil); Tríceps brachii (Paleta). In Vivo: PCA (Peso Corporal de Abate); COND (Condição Corporal). Carcaça: CONF (Conformação); EE (Estado de Engorduramento); EGS (Espessura de Gordura Subcutânea); AOL (Área de Olho de Lombo); ICC (Índice de Compacidade da Carcaça); GTO (Gordura Total - gordura subcutânea e intermuscular); MTO (Músculo Total); MG (Relação Músculo/Gordura Total); PCO (Porção Comestível - peso de músculo e gordura total). $\left({ }^{*} \mathrm{P}<0,05\right)$.

Tabela 6. Correlação entre as características in vivo e na carcaça com as análises centesimais dos músculos Longissimus Dorsi (LD), Bíceps Femoris (BF), Semimembranosus (SM), Tríceps Brachii (TB) de cordeiros Comerciais e Pantaneiros.

\begin{tabular}{|c|c|c|c|c|c|c|c|c|c|c|c|c|}
\hline \multicolumn{2}{|c|}{ Músculos/Cordeiros } & PCA & COND & CONF & EE & EGS & $\mathrm{AOL}$ & ICC & GTO & MTO & MG & $\mathrm{PCO}$ \\
\hline \multicolumn{13}{|c|}{ Proteína Bruta } \\
\hline \multirow[t]{2}{*}{ LD } & Comerciais & 0,16 & $-0,11$ & $-0,01$ & 0,08 & $-0,03$ & 0,29 & 0,09 & $-0,01$ & 0,13 & 0,08 & 0,08 \\
\hline & Pantaneiros & 0,12 & $-0,03$ & 0,03 & $-0,14$ & 0,20 & 0,04 & 0,30 & 0,20 & 0,09 & $-0,12$ & 0,18 \\
\hline \multirow[t]{2}{*}{$\mathrm{BF}$} & Comerciais & 0,10 & 0,22 & $-0,03$ & $-0,04$ & $-0,04$ & 0,26 & 0,07 & 0,05 & 0,07 & 0,00 & 0,07 \\
\hline & Pantaneiros & 0,02 & $-0,09$ & 0,10 & $-0,16$ & 0,03 & $0,38^{*}$ & 0,08 & $-0,04$ & 0,09 & 0,06 & 0,06 \\
\hline \multirow[t]{2}{*}{ SM } & Comerciais & 0,18 & 0,17 & 0,00 & $-0,04$ & 0,00 & 0,26 & 0,07 & 0,22 & $0,34^{*}$ & $-0,11$ & $0,34^{*}$ \\
\hline & Pantaneiros & $-0,16$ & $-0,32^{*}$ & $-0,21$ & $-0,27$ & $-0,18$ & 0,09 & 0,02 & $-0,08$ & $-0,45^{*}$ & $-0,20$ & $-0,43^{*}$ \\
\hline \multirow[t]{2}{*}{ TB } & Comerciais & 0,11 & $-0,04$ & $-0,03$ & 0,02 & 0,00 & 0,25 & 0,12 & $-0,06$ & $-0,01$ & 0,04 & $-0,04$ \\
\hline & Pantaneiros & $-0,35^{*}$ & $-0,13$ & $-0,16$ & 0,06 & 0,16 & 0,08 & $-0,08$ & $-0,08$ & $-0,20$ & $-0,11$ & $-0,22$ \\
\hline \multicolumn{13}{|c|}{ Extrato Etéreo } \\
\hline \multirow[t]{2}{*}{$\overline{\mathrm{LD}}$} & Comerciais & $-0,03$ & $-0,15$ & $-0,16$ & 0,05 & $-0,04$ & $-0,07$ & 0,03 & $-0,02$ & $-0,39$ & $-0,16$ & $\overline{-0,26}$ \\
\hline & Pantaneiros & $-0,15$ & 0,31 & 0,17 & $0,32^{*}$ & 0,09 & 0,04 & 0,00 & 0,22 & 0,16 & $-0,15$ & 0,25 \\
\hline \multirow[t]{2}{*}{$\mathrm{BF}$} & Comerciais & 0,05 & 0,02 & 0,04 & 0,14 & $-0,07$ & 0,02 & 0,06 & 0,28 & $-0,02$ & $-0,25$ & 0,13 \\
\hline & Pantaneiros & $-0,07$ & $-0,08$ & $-0,05$ & 0,04 & 0,05 & $0,34^{*}$ & $-0,17$ & $-0,01$ & 0,12 & 0,03 & 0,10 \\
\hline \multirow[t]{2}{*}{ SM } & Comerciais & 0,04 & 0,13 & $-0,15$ & $-0,03$ & $-0,14$ & $-0,15$ & $-0,12$ & 0,01 & $-0,24$ & $-0,21$ & $-0,15$ \\
\hline & Pantaneiros & 0,01 & $0,59^{*}$ & 0,22 & 0,50 * & 0,08 & $-0,05$ & $-0,02$ & 0,28 & 0,27 & $-0,12$ & $0,38^{*}$ \\
\hline \multirow[t]{2}{*}{ TB } & Comerciais & $-0,01$ & 0,00 & $-0,02$ & 0,17 & 0,16 & 0,01 & 0,09 & 0,18 & $-0,17$ & $-0,25$ & $-0,02$ \\
\hline & Pantaneiros & $-0,08$ & $-0,19$ & 0,24 & 0,07 & 0,50 * & 0,20 & 0,07 & $-0,05$ & 0,25 & 0,16 & 0,20 \\
\hline \multicolumn{13}{|c|}{ Umidade } \\
\hline \multirow[t]{2}{*}{ LD } & Comerciais & 0,03 & $-0,17$ & $-0,07$ & 0,00 & $-0,07$ & 0,17 & 0,02 & $-0,11$ & 0,02 & 0,11 & $-0,04$ \\
\hline & Pantaneiros & 0,26 & 0,27 & 0,31 & 0,18 & 0,10 & $-0,03$ & $-0,23$ & 0,15 & 0,27 & 0,02 & 0,31 \\
\hline $\mathrm{BF}$ & Comerciais & $-0,09$ & 0,06 & $-0,03$ & $-0,06$ & 0,07 & $-0,17$ & $-0,08$ & $-0,16$ & 0,02 & 0,06 & $-0,07$ \\
\hline
\end{tabular}

Agrarian, Dourados, v. 14, n. 52, p. 244-259, 2021. 
Pagrarian

\begin{tabular}{|c|c|c|c|c|c|c|c|c|c|c|c|c|}
\hline \multirow{3}{*}{ SM } & Pantaneiros & 0,09 & $0,37^{*}$ & 0,18 & 0,20 & 0,04 & $-0,40^{*}$ & $-0,39^{*}$ & 0,28 & $-0,22$ & $-0,36^{*}$ & $-0,05$ \\
\hline & Comerciais & $0,40^{*}$ & $-0,04$ & 0,17 & 0,28 & $-0,15$ & $-0,03$ & 0,23 & 0,04 & 0,07 & $-0,04$ & 0,07 \\
\hline & Pantaneiros & 0,12 & $0,52^{*}$ & $0,39^{*}$ & $0,48^{*}$ & 0,07 & 0,13 & $-0,18$ & 0,29 & $0,37^{*}$ & $-0,04$ & $0,47^{*}$ \\
\hline \multirow[t]{2}{*}{$\mathrm{TB}$} & Comerciais & 0,05 & $-0,17$ & $-0,07$ & 0,00 & $-0,08$ & 0,17 & 0,02 & $-0,10$ & 0,01 & 0,10 & $-0,04$ \\
\hline & Pantaneiros & 0,08 & 0,16 & $-0,05$ & 0,16 & $-0,04$ & $-0,62^{*}$ & $-0,02$ & $0,40^{*}$ & $-0,45^{*}$ & $-0,60^{*}$ & $-0,19$ \\
\hline \multicolumn{13}{|c|}{ Matéria Mineral } \\
\hline \multirow[t]{2}{*}{ LD } & Comerciais & 0,10 & $-0,09$ & 0,04 & 0,12 & 0,02 & 0,12 & 0,12 & $-0,02$ & $-0,05$ & $-0,05$ & $-0,04$ \\
\hline & Pantaneiros & $-0,11$ & 0,22 & 0,07 & 0,24 & $-0,08$ & 0,05 & 0,01 & 0,11 & 0,15 & $-0,03$ & 0,19 \\
\hline \multirow[t]{2}{*}{$\mathrm{BF}$} & Comerciais & 0,03 & 0,19 & $-0,12$ & $-0,09$ & $-0,08$ & 0,21 & $-0,01$ & $-0,07$ & $-0,08$ & $-0,03$ & $-0,09$ \\
\hline & Pantaneiros & 0,00 & $-0,23$ & $-0,26$ & $-0,21$ & $-0,02$ & $-0,26$ & 0,09 & 0,09 & $-0,42^{*}$ & $-0,32^{*}$ & $-0,32^{*}$ \\
\hline \multirow[t]{2}{*}{ SM } & Comerciais & 0,07 & $-0,03$ & 0,04 & 0,12 & 0,20 & $0,37^{*}$ & 0,21 & 0,17 & 0,23 & 0,02 & 0,24 \\
\hline & Pantaneiros & $-0,12$ & $-0,25$ & $-0,03$ & $-0,17$ & $-0,06$ & $0,35^{*}$ & $0,46^{\star}$ & $-0,11$ & $-0,05$ & 0,07 & $-0,10$ \\
\hline \multirow[t]{2}{*}{ TB } & Comerciais & 0,16 & $-0,06$ & $-0,06$ & 0,07 & $-0,02$ & 0,17 & 0,11 & $-0,04$ & 0,01 & 0,03 & $-0,01$ \\
\hline & Pantaneiros & $-0,09$ & $-0,33^{*}$ & $-0,18$ & 0,05 & $-0,05$ & 0,06 & $-0,01$ & 0,17 & $-0,40^{*}$ & $-0,33^{*}$ & $-0,26$ \\
\hline
\end{tabular}

In Vivo: PCA (Peso Corporal ao Abate); COND (Condição Corporal). Carcaça: CONF (Conformação); EE (Estado de Engorduramento); EGS (Espessura de Gordura Subcutânea); AOL (Área de Olho de Lombo); ICC (Índice de Compacidade de Carcaça); GTO (Gordura Total da Carcaça); MTO (Músculo Total da Carcaça); MG (Relação Músculo/Gordura na Carcaça); PCO (Porção Comestível = peso de músculo e gordura total)..( $\left.{ }^{\star}<0,05\right)$.

Tabela 7. Equações de regressão múltipla entre as características in vivo e na carcaça com as análises instrumentais dos músculos dos cordeiros Comerciais.

\begin{tabular}{|c|c|c|c|}
\hline Músculos & Equação & P-value & $\mathrm{R}^{2}$ \\
\hline \multicolumn{4}{|c|}{ pH Final } \\
\hline Longissimus dorsi & $5,409+0,145^{*}$ COND - 0,039 *EGS & 0,031 & 0,12 \\
\hline Semimembranosus & $6,137+0,118^{*} \mathrm{COND}+0,107^{*} \mathrm{CONF}-0,234^{\star} \mathrm{EE}-0,086^{\star} \mathrm{MTO}$ & 0,016 & 0,20 \\
\hline Tríceps brachii & $6,007-0,042 * E G S$ & 0,036 & 0,08 \\
\hline \multicolumn{4}{|c|}{ Cor $L^{*}$} \\
\hline Longissimus dorsi & $52,331-2,662^{*} \mathrm{COND}-7,874^{*} \mathrm{GTO}+2,892^{*} \mathrm{MTO}-2,913^{*} \mathrm{MG}$ & 0,001 & 0,32 \\
\hline Semimembranosus & $54,205-0,636^{\star} \mathrm{PCA}+2,627^{\star} \mathrm{EE}-1,066^{\star} \mathrm{EGS}-0,317^{\star} \mathrm{AOL}$ & 0,003 & 0,27 \\
\hline Tríceps brachii & $39,031-0,331^{*} \mathrm{AOL}-1,495^{\star} \mathrm{GTO}+1,494^{*} \mathrm{MTO}$ & 0,033 & 0,14 \\
\hline \multicolumn{4}{|c|}{ 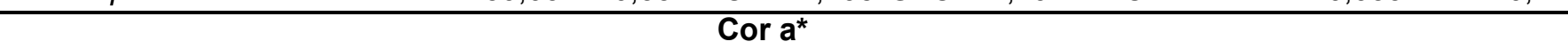 } \\
\hline Longissimus dorsi & $15,504+1,291^{*} \mathrm{EE}-1,778^{*} \mathrm{GTO}$ & 0,203 & 0,03 \\
\hline Semimembranosus & $15,806+1,412^{*}$ COND $-0,486^{*}$ EGS $-1,274^{*}$ GTO & 0,005 & 0,23 \\
\hline Tríceps brachii & $11,286-1,937^{*} \mathrm{EE}+42,041^{*} \mathrm{ICC}$ & 0,265 & 0,01 \\
\hline \multicolumn{4}{|c|}{${\text { Cor } b^{*}}^{*}$} \\
\hline Longissimus dorsi & $15,921-0,527^{*} \mathrm{PCA}-0,742^{*} \mathrm{EGS}+34,947^{*} \mathrm{ICC}$ & 0,069 & 0,10 \\
\hline Semimembranosus & $10,205+1,902^{*}$ COND $-0,646^{*}$ EGS $-28,084^{*} I C C$ & 0,002 & 0,27 \\
\hline Tríceps brachii & 5,670 & - & - \\
\hline \multicolumn{4}{|c|}{ Capacidade de Retenção de Agua } \\
\hline Longissimus dorsi & $57,160+0,462^{*} \mathrm{PCA}$ & 0,163 & 0,02 \\
\hline Bíceps femoris & $71,934+4,425^{\star} \mathrm{EE}-7,521^{*} \mathrm{GTO}$ & 0,049 & 0,10 \\
\hline Semimembranosus & $82,591-2,470 *$ MTO & 0,052 & 0,07 \\
\hline Tríceps brachii & $43,232-2,794^{\star} \mathrm{PCA}+395,01^{*} \mathrm{ICC}+4,335^{*} \mathrm{MG}$ & 0,014 & 0,18 \\
\hline \multicolumn{4}{|c|}{ Perda de Peso ao Cozimento } \\
\hline Longissimus dorsi & $97,220-0,838^{*} \mathrm{AOL}+3,596^{*} \mathrm{MTO}$ & 0,025 & 0,13 \\
\hline Bíceps femoris & 93,445 & - & - \\
\hline Semimembranosus & $58,851-8,183^{*} \mathrm{COND}+2,614^{*} \mathrm{EGS}$ & 0,011 & 0,17 \\
\hline Tríceps brachii & $42,495+2,670^{*} \mathrm{CONF}-90,628^{*} \mathrm{ICC}+3,349^{*} \mathrm{MTO}$ & 0,002 & 0,26 \\
\hline \multicolumn{4}{|c|}{ Força de Cisalhamento } \\
\hline Longissimus dorsi & $4,766-29,082^{*} \mathrm{ICC}+4,080^{*} \mathrm{GTO}+0,742^{*} \mathrm{MG}$ & 0,028 & 0,15 \\
\hline Bíceps femoris & $1,408+1,009^{*} \mathrm{COND}-0,138^{*} \mathrm{AOL}-1,016^{*} \mathrm{GTO}+0,565^{*} \mathrm{MTO}$ & 0,074 & 0,12 \\
\hline Semimembranosus & $9,145+1,183^{*}$ CONF $-27,822^{*}$ ICC & 0,164 & 0,04 \\
\hline Tríceps brachii & $6,437-0,089^{*} \mathrm{PCA}-0,358^{*} \mathrm{MG}$ & 0,025 & 0,13 \\
\hline
\end{tabular}

Agrarian, Dourados, v. 14, n. 52, p. 244-259, 2021.

Esta obra está licenciada com uma Licença Creative Commons Atribuição-NãoComercial-CompartilhaIqual 3.0 Brasil. 


\section{Pagrarian}

\section{$\therefore$ ain}

Músculos: Longissimus dorsi (Lombo); Semimembranosu e Bíceps femoris (Pernil); Tríceps brachii (Paleta). In Vivo: PCA (Peso Corporal de Abate); COND (Condição Corporal). Carcaça: CONF (Conformação); EE (Estado de Engorduramento); EGS (Espessura de Gordura Subcutânea); AOL (Área de Olho de Lombo); ICC (Índice de Compacidade da Carcaça); GTO (Gordura Total - gordura subcutânea e intermuscular); MTO (Músculo Total); MG (Relação Músculo/Gordura Total); PCO (Porção Comestível - peso de músculo e gordura total).

Tabela 8. Equações de regressão múltipla entre as características in vivo e na carcaça com as análises instrumentais dos músculos dos cordeiros Pantaneiros.

\begin{tabular}{|c|c|c|c|}
\hline Músculos & Equação & P-value & $\mathrm{R}^{2}$ \\
\hline \multicolumn{4}{|c|}{ pH Final } \\
\hline Longissimus dorsi & $5,723+0,019^{*} \mathrm{PCA}-0,236^{*} \mathrm{COND}+0,160^{*} \mathrm{CONF}-0,162^{*} \mathrm{MTO}+$ & 0,002 & 0,31 \\
\hline Semimembranosus & $6,157-0,021^{*} \mathrm{PCA}-0,146^{*} \mathrm{COND}+3,763^{*} \mathrm{ICC}-0,075^{\star} \mathrm{MTO}$ & 0,003 & 0,27 \\
\hline Tríceps brachii & $6,357-0,083^{*} \mathrm{COND}-0,020^{*} \mathrm{AOL}$ & 0,045 & 0,10 \\
\hline \multicolumn{4}{|c|}{ Cor $L^{*}$} \\
\hline Longissimus dorsi & $38,943+0,304^{*} \mathrm{PCA}+1,319^{*} \mathrm{COND}+0,589^{*} \mathrm{AOL}-6,865^{*} \mathrm{GTO}-$ & 0,001 & 0,33 \\
\hline Semimembranosus & $30,017+2,309^{*} \mathrm{COND}-1,786^{*} \mathrm{EGS}+0,734^{*} \mathrm{AOL}-1,473^{*} \mathrm{MG}$ & 0,001 & 0,33 \\
\hline Tríceps brachii & $40,597+2,350^{*}$ COND $-2,111^{*} \mathrm{CONF}$ & 0,133 & 0,05 \\
\hline \multicolumn{4}{|c|}{ Cor $\mathbf{a}^{*}$} \\
\hline Longissimus dorsi & $28,481+0,276^{*} \mathrm{PCA}+2,375^{*} \mathrm{CONF}-0,679^{*} \mathrm{EGS}-59,734^{*} \mathrm{ICC}-$ & $<0,001$ & 0,45 \\
\hline Semimembranosus & $15,680+0,301^{*} \mathrm{PCA}+1,676^{*} \mathrm{CONF}+0,504^{*} \mathrm{AOL}-3,828^{*} \mathrm{MTO}$ & 0,0001 & 0,41 \\
\hline Tríceps brachii & $20,036+0,457^{*} \mathrm{PCA}+2,732^{*} \mathrm{CONF}-61,360^{*} \mathrm{ICC}-2,193^{*} \mathrm{MG}$ & 0,002 & 0,29 \\
\hline \multicolumn{4}{|c|}{ Cor $b^{*}$} \\
\hline Longissimus dorsi & $11,354-1,373^{*} \mathrm{COND}+2,211^{*} \mathrm{CONF}-1,284^{*} \mathrm{EE}+0,417^{*} \mathrm{EGS}+$ & $<0,001$ & 0,57 \\
\hline Semimembranosus & $9,146+0,374^{\star} \mathrm{PCA}-2,288^{\star} \mathrm{EE}+0,807^{\star} \mathrm{EGS}+0,305^{\star} \mathrm{AOL}-$ & $<0,001$ & 0,50 \\
\hline Tríceps brachii & $13,126-1,410^{\star} \mathrm{COND}+0,781^{*} \mathrm{EGS}-1,215^{\star} \mathrm{MG}$ & 0,002 & 0,26 \\
\hline \multicolumn{4}{|c|}{ Capacidade de Retenção de Água } \\
\hline Longissimus dorsi & $106,85-3,219^{*} \mathrm{CONF}-51,7111^{*} \mathrm{ICC}+2,790^{*} \mathrm{MTO}-4,315^{*} \mathrm{MG}$ & 0,020 & 0,19 \\
\hline Bíceps femoris & $74,108+3,870^{\star} \mathrm{EE}-2,932^{\star} \mathrm{EGS}+1,144^{*} \mathrm{AOL}-41,048^{*} \mathrm{ICC}$ & $<0,0001$ & 0,46 \\
\hline Semimembranosus & $24,267+0,502^{*} \mathrm{PCA}+5,148^{*} \mathrm{CONF}-3,522^{*} \mathrm{EE}+2,450^{*} \mathrm{EGS}-$ & $<0,0001$ & 0,45 \\
\hline Tríceps brachii & $99,281+7,660^{*} \mathrm{GTO}-5,868^{*} \mathrm{MTO}$ & 0,0105 & 0,17 \\
\hline \multicolumn{4}{|c|}{ Perda de Peso ao Cozimento } \\
\hline Longissimus dorsi & $40,348-4,072^{*} \mathrm{COND}+5,658^{*} \mathrm{CONF}-8,880^{*} \mathrm{GTO}$ & 0,0001 & 0,29 \\
\hline Bíceps femoris & $36,221-2,654^{*} \mathrm{MTO}+2,394^{*} \mathrm{MG}$ & 0,201 & 0,03 \\
\hline Semimembranosus & $24,267+0,502^{*} \mathrm{PCA}+5,148^{*} \mathrm{CONF}-3,522^{*} \mathrm{EE}+2,450^{*} \mathrm{EGS}-$ & $<0,0001$ & 0,63 \\
\hline Tríceps brachii & $48,867-0,772^{*} \mathrm{PCA}-8,604^{*} \mathrm{EE}+1,863^{*} \mathrm{EGS}+111,793^{*} \mathrm{ICC}$ & 0,0003 & 0,37 \\
\hline \multicolumn{4}{|c|}{ Força de Cisalhamento } \\
\hline Longissimus dorsi & $7,087+0,741^{*} \mathrm{CONF}-1,160^{*} \mathrm{EE}-0,719^{*} \mathrm{MG}$ & 0,0394 & 0,13 \\
\hline Bíceps femoris & 2,227 & - & - \\
\hline Semimembranosus & $10,460-0,127^{\star} \mathrm{PCA}-0,797^{\star} \mathrm{EE}-0,136^{*} \mathrm{AOL}-22,469^{*} \mathrm{ICC}+$ & 0,0023 & 0,34 \\
\hline Tríceps brachii & $2,865+0,793^{*} \mathrm{COND}-0,588^{\star} \mathrm{CONF}+0,352^{\star} \mathrm{EE}-0,395^{\star} \mathrm{MTO}$ & 0,0003 & 0,28 \\
\hline
\end{tabular}

Músculos: Longissimus dorsi (Lombo); Semimembranosu e Bíceps femoris (Pernil); Tríceps brachii (Paleta). In Vivo: PCA (Peso Corporal de Abate); COND (Condição Corporal). Carcaça: CONF (Conformação); EE (Estado de Engorduramento); EGS (Espessura de Gordura Subcutânea); AOL (Área de Olho de Lombo); ICC (Índice de Compacidade da Carcaça); GTO (Gordura Total - gordura subcutânea e intermuscular); MTO (Músculo Total); MG (Relação Músculo/Gordura Total); PCO (Porção Comestível - peso de músculo e gordura total). 
Tabela 9. Equações de regressão múltipla entre as características in vivo e na carcaça com as análises centesimais dos músculos Longissimus dorsi (LD), Bíceps femoris (BF), Semimembranosus (SM) e Tríceps Brachii (TB) de cordeiros Comerciais e Pantaneiros.

\begin{tabular}{|c|c|c|c|c|}
\hline \multirow{2}{*}{\multicolumn{2}{|c|}{ Músculos/Cordeiros }} & Equação & P-value & $\mathrm{R}^{2}$ \\
\hline & & Proteína Bruta & & \\
\hline \multirow[t]{2}{*}{ LD } & Comerciais & $17,811-2,355^{*} \mathrm{COND}+0,648^{*} \mathrm{AOL}$ & 0,033 & 0,12 \\
\hline & Pantaneiros & $20,130-2,882^{*} \mathrm{EE}+0,835^{\star} \mathrm{EGS}+4,296^{\star} \mathrm{GTO}$ & 0,0003 & 0,35 \\
\hline \multirow[t]{2}{*}{$\mathrm{BF}$} & Comerciais & $13,484+0,345^{\star} \mathrm{AOL}$ & 0,106 & 0,04 \\
\hline & Pantaneiros & $12,590+0,909^{*} \mathrm{CONF}-0,840^{*} \mathrm{EE}+0,438^{*} \mathrm{AOL}$ & 0,0238 & 0,16 \\
\hline \multirow[t]{2}{*}{ SM } & Comerciais & $17,257+0,159^{\star} \mathrm{PCA}-0,921^{*} \mathrm{COND}-2,418^{*} \mathrm{EE}+0,200^{*} \mathrm{AOL}+35,562^{*} \mathrm{ICC}$ & 0,005 & 0,28 \\
\hline & Pantaneiros & $21,960+49,232^{*} \mathrm{ICC}-2,622^{*} \mathrm{GTO}-3,030^{*} \mathrm{MTO}$ & 0,0013 & 0,29 \\
\hline \multirow[t]{2}{*}{ TB } & Comerciais & $10,848-1,753^{*} \mathrm{COND}-1,887^{*} \mathrm{CONF}+0,573^{*} \mathrm{AOL}+35,562^{*} \mathrm{ICC}$ & 0,151 & 0,07 \\
\hline & Pantaneiros & $24,293-0,167^{*} \mathrm{PCA}-0,643^{*} \mathrm{CONF}+25,749^{*} \mathrm{ICC}-3,711^{*} \mathrm{GTO}-1,207^{*} \mathrm{MG}$ & 0,0121 & 0,27 \\
\hline & & Extrato Etéreo & & \\
\hline \multirow{2}{*}{$\overline{L D}$} & Comerciais & $9,728-1,005^{*} \mathrm{CONF}+0,193^{*} \mathrm{AOL}+1,495^{*} \mathrm{GTO}-1,935^{*} \mathrm{MTO}$ & 0,043 & 0,15 \\
\hline & Pantaneiros & $10,207-0,403^{*} \mathrm{PCA}+1,140^{*} \mathrm{COND}+2,039^{*} \mathrm{MTO}-1,225^{*} \mathrm{MG}$ & 0,0099 & 0,22 \\
\hline \multirow[t]{2}{*}{$\mathrm{BF}$} & Comerciais & $6,151-0,441^{\star} \mathrm{EGS}-18,991^{\star} \mathrm{ICC}+4,699^{\star} \mathrm{GTO}-1,519^{\star} \mathrm{MTO}+0,645^{\star} \mathrm{MG}$ & 0,021 & 0,21 \\
\hline & Pantaneiros & $1,942+0,337^{\star} \mathrm{AOL}-14,168^{*} \mathrm{ICC}+0,673^{\star} \mathrm{MTO}$ & 0,0618 & 0,11 \\
\hline \multirow[t]{2}{*}{ SM } & Comerciais & $7,223+0,128^{*} \mathrm{PCA}-25,198^{*} \mathrm{ICC}-0,480^{*} \mathrm{MG}$ & 0,025 & 0,16 \\
\hline & Pantaneiros & $8,032-0,312^{*} \mathrm{PCA}+3,178^{*} \mathrm{COND}-1,167^{*} \mathrm{CONF}-0,635^{*} \mathrm{EGS}$ & 0,001 & 0,48 \\
\hline \multirow[t]{2}{*}{ TB } & Comerciais & $7,522+1,625^{*} \mathrm{GTO}-2,248^{*} \mathrm{MTO}$ & 0,117 & 0,06 \\
\hline & Pantaneiros & $6,055-1,901^{*} \mathrm{COND}+1,408^{*} \mathrm{CONF}+0,890^{*} \mathrm{EGS}$ & $<0,0001$ & 0,40 \\
\hline & & Umidade & & \\
\hline & Comerciais & $73,114+7,919^{*} \mathrm{COND}+1,630^{*} \mathrm{AOL}$ & 0,105 & 0,06 \\
\hline LD & Pantaneiros & $77,501+0,224^{*} \mathrm{PCA}-0,391^{*} \mathrm{AOL}-34,220^{*} \mathrm{ICC}+1,682^{*} \mathrm{MTO}-1,048^{*} \mathrm{MG}$ & 0,066 & 0,14 \\
\hline \multirow[t]{2}{*}{$\mathrm{BF}$} & Comerciais & $73,934+1,066^{\star} \mathrm{EE}+0,506^{\star} \mathrm{EGS}-0,157^{\star} \mathrm{AOL}-5,314^{*} \mathrm{GTO}+2,080^{\star} \mathrm{MTO}$ & 0,033 & 0,20 \\
\hline & Pantaneiros & $79,893+1,904^{*} \mathrm{COND}-0,831^{*} \mathrm{EGS}-0,469^{*} \mathrm{AOL}-0,888^{*} \mathrm{MG}$ & $<0,0001$ & 0,49 \\
\hline \multirow[t]{2}{*}{ SM } & Comerciais & $55,643+0,448^{\star} \mathrm{PCA}+3,684^{*} \mathrm{EE}-4,750^{*} \mathrm{GTO}$ & 0,007 & 0,22 \\
\hline & Pantaneiros & $64,997+3,386^{*} \mathrm{EE}-1,170^{*} \mathrm{EGS}-37,698^{*} \mathrm{ICC}+3,185^{*} \mathrm{MTO}$ & 0,0002 & 0,38 \\
\hline \multirow[t]{2}{*}{ TB } & Comerciais & $73,452-8,080^{*} \mathrm{COND}+1,665^{\star} \mathrm{AOL}$ & 0,098 & 0,07 \\
\hline & Pantaneiros & $79,597+0,320^{*} \mathrm{PCA}+1,759^{*} \mathrm{COND}-1,917^{*} \mathrm{EGS}-36,807^{\star} \mathrm{ICC}-2,319^{*} \mathrm{MG}$ & $<0,06$ & 0,70 \\
\hline & & Matéria Mineral & & \\
\hline & Comerciais & 0,882 & - & - \\
\hline LD & Pantaneiros & $1,544-0,056^{*} \mathrm{PCA}+0,267^{\star} \mathrm{EE}-0,111^{*} \mathrm{EGS}+0,291^{*} \mathrm{MTO}$ & 0,120 & 0,09 \\
\hline \multirow[t]{2}{*}{ BF } & Comerciais & $0,847-0,095^{\star} \mathrm{CONF}+0,026^{*} \mathrm{AOL}$ & 0,115 & 0,06 \\
\hline & Pantaneiros & $2,921-0,292^{\star} \mathrm{EE}-0,272^{\star} \mathrm{MG}$ & 0,0026 & 0,23 \\
\hline \multirow[t]{2}{*}{ SM } & Comerciais & $0,064-0,223^{*} \mathrm{COND}-0,197^{\star} \mathrm{CONF}+0,056^{*} \mathrm{EGS}+0,071^{*} \mathrm{AOL}+3,179^{*} \mathrm{ICC}$ & 0,007 & 0,26 \\
\hline & Pantaneiros & $1,187-0,146^{\star} \mathrm{EE}+0,117^{\star} \mathrm{EGS}+0,049^{\star} \mathrm{AOL}-0,085^{\star} \mathrm{MTO}$ & 0,0005 & 0,36 \\
\hline \multirow{2}{*}{ TB } & Comerciais & 0,879 & - & - \\
\hline & Pantaneiros & $1,622+0,011^{\star} \mathrm{PCA}-0,121^{*} \mathrm{COND}+0,057^{\star} \mathrm{EGS}-0,118^{*} \mathrm{AOL}+0,087^{*} \mathrm{GTO}$ & 0,0035 & 0,32 \\
\hline & 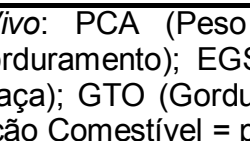 & $\begin{array}{l}\text { Oondição Corporal). Carcaça: CONF (Conformaçâ } \\
\text { nea); AOL (Área de Olho de Lombo); ICC (Índice } \\
\text { ulo Total da Carcaça); MG (Relação Músculo/Gordu } \\
<0,05 \text { ). }\end{array}$ & $\begin{array}{l}\text { EE (Es } \\
\text { Compac }\end{array}$ & $\begin{array}{ll}\text { do de } \\
\text { ade de }\end{array}$ \\
\hline
\end{tabular}

\section{Conclusão}

As características in vivo e na carcaça apresentaram baixa correlação e não foram eficientes para predizer as características dos músculos dos cordeiros Comerciais e Pantaneiros.

\section{Agradecimentos}

À CAPES pela concessão da bolsa. À CAPES, CNPq e UFGD pelo financiamento do projeto (PVE edital 061-2012). Aos membros dos grupos de pesquisas Ovinotecnia e o de Carcaças e Carnes da UFGD pela colaboração e execução do projeto. 


\section{Referências}

AOAC - Association of Official Analytical Chemists. Official methods of analysis of the AOAC International. (2005). $18^{\mathrm{a}}$ ed. Gaithersburg.

BRASIL. Decreto $n^{\circ}$. 30.691, de 29 de março de 1952. Regulamento de Inspeção Industrial e Sanitária de Produtos de Origem Animal (RIISPOA). Ministério da Agricultura Pecuária e Abastecimento. Diário Oficial da República Federativa do Brasil, Brasília, 1952.

BRASIL. Instrução Normativa n.3, janeiro de 2000. Ministério da Agricultura. Regulamento técnico de métodos de insensibilização para o abate humanitário de animais de açougue. S.D.A./M.A.A. Diário Oficial da União, Brasília, 2000.

Cañeque, V., Sañudo, C. (2000) Metodologia para el estúdio de la calidad de la canal y de la carne em ruminantes. INIA, Madrid, 254p.

Cartaxo, F.Q., Sousa, W.H., Cezar, M.F., Cunha, M.G.G., Menezes, I.M., Ramos J.P.F., Gomes, J.T., Viana, J. A. (2017). Desempenho e características de carcaça de cordeiros Santa Inês e suas cruzas com Dorper terminados em confinamento. Revista Brasileira de Saúde e Produção Animal.v.18, n.02, p.388401. https://doi.org/10.1590/S1519-99402017000200017

Cirne, L.G.A., Oliveira, G.J.C., Jaeger, S.M.P.I., Bagaldo, A.R., Leite, M.C.P., Rocha, N.N., Macedo Junior, C. M., Oliveira, P.A. (2014). Comportamento ingestivo de cordeiros em confinamento, alimentados com dieta exclusiva de concentrado com diferentes porcentagens de proteína. Arquivo Brasileiro de Medicina Veterinária e Zootecnia. v.66, n.01, p.229-234. https://doi.org/10.1590/S0102-09352014000100031

Cruz, B.C.C., Santos, C.L., Azevedo, J.A., Silva, D.A. (2016). Avaliação e composição centesimal e as características físico-químicas da carne de ovinos. Pubvet. v.10, n.02, p.147-162. DOI: 10.22256/pubvet.v10n2.147-162

Ferreira, M.B., Guidolin, D.G.F., Vargas Junior, F.M., Reis, F.A., Costa, J.A.A., Dal-Ponte Vieira, A.C.R. (2020). Embrapa Caprinos e Ovinos - Capítulo em livro técnico (INFOTECA -E). Grupo Genético Pantaneiro. Cap.9, p.163 - 173

Gomide, L.A.M., Ramos, E.M., Fontes, P.R. (2013) Ciência e qualidade da Carne - Fundamentos. Viçosa: ed. UFV, 197p.

Lambe, N.R., Navajas, E.A., Bunger, L., Fisher, A.V., Roehe, R., Simm, G. (2009) Prediction of lamb carcass composition and meat quality using combinations of post-mortem measurements. Meat Science. v.81, p.711719. https://doi.org/10.1016/j.meatsci.2008.10.025

Menezes, B.B., Ribeiro, C.B., Walker, C.C., Melo, G.K.A., Souza, A.R.D.L., Fernandes, H.J., Franco, G.L., Morais, M.M. (2015) Predição da composição física e química da carcaça de borregas pela seção da $9^{a}$ a $11^{a}$ costelas ou $12^{\mathrm{a}}$ costela. Revista Brasileira de Saúde e Produção Animal. v.16, n.4, p.874884. https://doi.org/10.1590/S1519-99402015000400011

Osório, J.C.S., Osório, M.T.M. (2005) Produção de carne ovina: Técnicas de avaliação "in vivo" e na carcaça. $2^{\mathrm{a}}$ ed. Pelotas, 82p.

Osório, M.T.M., Osório, J.C.S., Silva Sobrinho, A.G. (2008). Avaliação instrumental da carne ovina. In: Silva Sobrinho, A.G., Sañudo, C., Osório, J.C.S., Arribas, M.M.C., Osório, M.T.M. Produção de carne ovina, Jaboticabal, Funep, 228p.

Osório, J.C.S., Osório, M.T.M., Sañudo, C. (2009). Características sensoriais da carne ovina. Revista Brasileira de Zootecnia. v.38, p.292-300. DOI: 10.1590/S1516-35982009001300029

Osório, J.C.S., Osório, M.T.M., Fernandes, A.R.M., Vargas Junior, F.M. (2014). Produção e qualidade de carne ovina. In: Selaive-Villaroel, A.B.; Osório, J.C.S. Produção de ovinos no Brasil. 1. ed, São Paulo: Roca, cap.28, p.399-445

Pereira, L.C., Ítavo, L.C.V., Mateus, R.G., Silva, D.C.G., Ferrreira, M.B., Carvalho, C.M. E. (2016). Análise econômica da alimentação de cordeiros confinados pela substituição parcial de concentrado pela parte aérea da mandioca. Acta Veterinaria Brasilica, v.10, n.3, p.258-265 


\section{PAGRARIAL}

Rego, F.C.A., Lima, L.D., Gasparini, M.J., Eleodoro, J.I., Zundth, M. (2019). Desempenho, características da carcaça e da carne de cordeiros confinados com níveis crescentes de bagaço de laranja em substituição ao milho. Ciência Animal Brasileira. v.20, p.01-12. https://doi.org/10.1590/1809-6891v20e-50159

Santos, C.L., Pérez, J.R.O., Cruz, C.A.C., Muniz, J.A., Santos, I.P.A., Almeida, T.R.V. (2008). Análise centesimal dos cortes da carcaça de cordeiros Santa Inês e Bergamácia. Ciência e Tecnologia de Alimentos. v.28, p.51-59. https://doi.org/10.1590/S0101-20612008000100009

Sañudo, C., Campo, M.M., Sierra, I., María, G.A., Olleta, J.L., Santolaria, P. (1997). Breed effect on carcass and meat quality of suckling lambs. Meat Science. v.46, n.04, p.357-365

Sañudo, C., Guerrero, A., Magalhães, D. (2017) Importância e necessidade das marcas de qualidade na carne e produtos cárneos (passado, presente e futuro). Guia Prático: Marcas de Carne e Produtos Cárneos. In: Texeira, A. Red CYTED. MARCARNE. Instituto Politécnico de Bragança, Portugal. v.01, p.05-16

Simões, J.A., Ricardo, R. (2000). Avaliação da cor da carne tomando como referência o músculo rectus abdominis, em carcaças de borregos leves. Revista Portuguesa de Ciências Veterinárias. v.95, n.535, p.124127. 